\title{
NIVELES DE IDENTIDAD EN ESCOLARES DE CLASE MEDIA BAJA
}

\author{
Lupe García Ampudia \\ Oswaldo Orellana Manrique \\ Pedro García Pizarro \\ Manuel Vega Gonzales \\ Manuel Torres Valladares
}

\begin{abstract}
RESUMEN
Se trabajó con escolares de cuarto y quinto de secundaria, procedentes de clase media baja de cuatro centros educativos pertenecientes al cono este y norte para analizar las características de identidad en sus niveles: nacional, generacional, regional, de género, familiar y personal.

Para la recolección de datos se elaboró un instrumento basado en el inventario de identidad de Zavalloni que establece la relación entre la conciencia subjetiva y los atributos sociales objetivos, las respuestas de los sujetos fueron codificadas con sus respectivas frecuencias y clasificadas en función de su orientación positiva, negativa o neutra, finalmente las respuestas positivas y negativas fueron clasificadas en cinco categorías: instrumentales, afectivas, sociales, morales y políticas.

Los resultados indicaron la presencia predominante de las respuestas instrumentales y la orientación positiva de las respuestas en cada uno de los niveles, siendo más evidentes las referidas a la identidad generacional y familiar.
\end{abstract}

\section{Palabras clave}

Identidad, globalización, mismidad, cultura subjetiva, percepción social.

\begin{abstract}
One worked with school of room and recruit of secondary, coming from low middle class of four educational centers belonging to the cone this and north to analyze the characteristics of identity in their levels: national, generational, regional, of gender, relative and personnel.

For the gathering of the data an instrument was elaborated based on the inventory of identity of Zavalloni that establishes the relationship between the subjective science and the attributes social objectives, the answers of the fellows they were coded with its respective frequencies and classified in function of its positive orientation, negative or neuter, finally the positive answers and negatives were classified in five categories: instrumental, affective, social, moral and polítical.

The results indicated the predominant presence of the instrumental answers and the positive orientation of the answers in each one of the levels, being more evident those referred to the identity generational and family.
\end{abstract}

\section{Key words}

Identity, globalización, mismidad, subjective culture, social perception. 


\section{INTRODUCCIÓN}

Uno de los problemas que recorre toda nuestra historia desde la época de la colonia hasta la actualidad, es el de la identidad. La presencia española en nuestro país con toda la violencia que significó el casi exterminio de la población indígena, también penetró en la cultura y la vida de las personas, desequilibrando no sólo el mundo social sino también el mundo personal donde los referentes fundamentales pretendieron ser sustituidos por otros considerados superiores.

Desde entonces, las interrogantes acerca de quienes somos, qué es el Perú, ¿quien soy? tiene respuestas que evidencian conflictos, y que van desde la negación de si mismos acompañados de la vergüenza de ser como somos hasta el desprecio y desvalorización del país y su historia.

Los cambios acelerados que se han producido en las últimas décadas a nivel mundial y la era de la globalización en que vivimos han traído nuevamente al debate el tema de la identidad, es así que muchos entusiastas sostienen que la globalización ha roto todas las fronteras y es obsoleto pensar en términos nacionales y de identidades formadas a su alrededor; olvidando que no puede haber un «nosotros universal» si no se construye los «nosotros particulares».

Sin embargo es importante señalar que en la época actual se han dado avances importantes en el abordaje teórico de la identidad, entendiendo que si el Perú es un país pluricultural y multirracial, la única vía posible de construir una identidad nacional es reconociendo y respetando las diferencias sin pretender hegemonías de ninguna de ellas.

La psicología social en Latinoamérica no ha sido ajena a la investigación de la identidad, es así que los psicólogos venezolanos (M. Montero, J. Salazar, E. Santoro) el mexicano Díaz Guerrero y los cubanos ( C. La Torre y F. Gonzales) son pioneros en la investigación sobre el tema, en el Perú la investigación ha sido más productiva en las ciencias sociales que en la psicología.

La presente investigación se orientó a identificar las características de la identidad en escolares de cuarto y quinto de secundaria y de procedencia media baja, por considerar que es un grupo etáreo que ha nacido con el impacto de una serie de hechos como el de la globalización y la violencia política en nuestro país.

En la identidad se consideró los niveles: nacional, generacional (la muestra es de jóvenes) género (masculino y femenino) regional (sólo se consideró a los limeños) familiar y personal, planteándonos las siguientes interrogantes:

¿Cuáles son las características de la identidad (en sus niveles nacional, regional, generacional, de género, familiar y personal) en estudiantes secundarios de clase media baja?

¿Cuáles son las categorías predominantes de los diferentes niveles de identidad: nacional, regional, generacional, de género, familiar y personal?

¿Es la orientación positiva o negativa la predominante en los diferentes niveles de la identidad? 
En la revisión bibliográfica sobre identidad se puede identificar varios modelos explicatorios de la misma, entre ellos tenemos los siguientes:

\section{- El Modelo Empírico-Metodológico}

De acuerdo con este modelo, la identidad social (identidad psicosocial o medio ambiente interior operativo) estaría constituida por núcleos dinámicos sociomotivacionales en los cuales se producen las reglas de recodificación de la realidad. Por lo tanto, tal identidad vendría a ser una construcción psíquica del mundo. El individuo es visto como situado en una matriz social constituida por la identidad social de si mismo (nacionalidad, etnia, región de origen, sexo, edad, clase social, estado civil, profesión u ocupación principal, religión o creencia religiosa, preferencia política, etc.) y la identidad social del "alter" siendo el alter equivalente a los individuos o colectividades de otra cultura, etnia, religión, clase social, sexo opuesto, etc.

\section{- El Modelo psicológico de la Personalidad y Cultura.}

Se caracteriza porque el objetivo fundamental es buscar describir los rasgos de personalidad característicos de los individuos que comparten una cultura.

Las búsquedas orientadas alrededor de los conceptos de cultura subjetiva (Triandis) y de representación social (Moscovici) serían clasificables también dentro de esta agrupación general.

\section{- El Modelo Lógico del principio de identidad.}

Analiza el problema de identidad social en función del principio de identidad desde la perspectiva de la lógica.

Ese principio anuncia que toda cosa es idéntica a si misma (solo puede ser ella y no su contraria), y es complementado por el de exclusión, se incluye a sí misma y excluye todo lo que no es ella; y por el principio de finalidad (toda conducta debe responder a un fin).

Para este modelo, el dominio occidental se fundamenta en esos postulados. Se define quien es y qué es, se niega la existencia a quienes no son y se atribuye una finalidad legitimadora a dicha conducta.

\section{- El Modelo Subjetivista.}

Este enfoque tiene que ver con la forma como los integrantes de un grupo se experiencian a sí mismos, es decir se refiere más al si mismo que a la descripción externa de los individuos. Esta es la línea que se deriva de la posición adoptada del sujeto de una "identidad social" que comienza con la existencia y aceptación de una etiqueta.

Éste modelo se interesa más en como los miembros del grupo se conciben a sí mismos, que consideramos es mas importante en el momento de la acción. Esta línea de trabajo es mucho mas nueva, pero puede nutrirse de trabajos realizados acerca de estereotipos y aut estereotipos, que también es una forma subjetivista de enfocar el problema.

Sintetizando los postulados los diferentes modelos podemos afirmar que el ser humano adquiere su identidad en interacción con los demás y como resultado de su experiencia vital, en ésta interacción se puede observar cómo lo social se va transformando en individual, en el que se mezcla lo objetivo y lo subjetivo que es lo que finalmente confiere a la identidad un carácter complejo y múltiple y en muchos casos contradictorio cuando 
entran en conflicto los diferentes niveles sean éstos personales, familiares, generacionales etc.

La identidad a su vez implica conciencia de la mismidad, construida en un proceso de diferenciación e identificación con el otro; es así que la identidad, tanto personal como social, en un sentido positivo implica conocimiento, sentimientos de pertenencia, compromiso, orgullo y acción compartida y tiene componentes cognitivos, afectivos y comportamentales sin los cuales no puede entenderse.

Los objetivos que nos planteamos son los siguientes:

1. Identificar las características de la identidad en escolares de nivel socioeconómico medio bajo.

2. Identificar las categorías predominantes en los diferentes niveles de la identidad.

3. Comparar las características de la identidad en sus diferentes niveles: nacional, regional, generacional, de género, familiar y personal

4. Identificar la dirección positiva y negativa de la identidad en sus diferentes niveles.

\section{MÉTODO}

\section{Sujetos}

Se consideró 200 estudiantes de cuarto y quinto de secundaria de cuatro centros educativos correspondientes a zonas urbano marginales del cono Este y norte. De los 200 estudiantes, 100 correspondieron al sexo femenino y 100 al sexo masculino y cuyas edades fluctuaron entre los 16 y 17 años.

\section{Instrumentos}

Para la evaluación de la identidad en sus diferentes niveles se procedió a elaborar un instrumento de carácter abierto basado en el inventario de identidad social de Zavalloni, que permite relacionar la conciencia subjetiva de la identidad con los atributos sociales objetivos de la misma, para ello se solicitó a los sujetos que escribieran las cinco primeras ideas que se les viene a la mente cuando evocan a determinado grupo de personas y a si mismos; para lo cual se consideró las siguientes frases abiertas:

Nosotros los Peruanos (identidad nacional)

Nosotros los jóvenes (identidad generacional)

Nosotras las mujeres (identidad de género)

Nosotros los hombres (identidad de género)

Nosotros los Limeños (identidad regional)

Nosotros los de mi familia (identidad familiar), y

Yo soy (identidad personal).

\section{Procedimientos}

Para el análisis de los resultados se procedió al registro de todas las respuestas de los sujetos a cada una de las frases, se codificó las mismas con su respectiva frecuencia, luego se procedió a clasificar las respuestas en función de su orientación positiva, negativa o neu- 
tra, se calificaron como neutras las res puestas descriptivas como extrovertido, mestizo etc.

Finalmente se procedió a clasificar en cinco categorías las respuestas positivas y negativas de los sujetos, éstas categorías fueron cinco: Instrumental, social, afectiva, moral y política.

\section{RESULTADOS Y DISCUSIÓN}

En la tabla $\mathrm{N}^{\circ} 1$ se observa los resultados de cada uno de los niveles de identidad considerando las cinco categorías mencionadas. Es así que se observa un predominio de respuestas agrupadas en la categoría instrumental, y una presencia insignificante de respuestas en la categoría política, que a su vez solamente está presente en la dimensión nacional, regional y familiar y ausente en las demás dimensiones.

En el nivel nacional es interesante señalar que las dos categorías predominantes son señalar que las dos categorías predominantes son las instrumentales y morales, siendo insignificante la presencia de la categoría afectiva y política.

Tabla $\mathbf{N}^{\circ} 1$

Distribución de respuestas por categorías y niveles de identidad en porcentajes

\begin{tabular}{|l|c|c|c|c|c|}
\hline \multirow{2}{*}{ Nivel Identidad } & \multicolumn{5}{|c|}{ Categoría } \\
\cline { 2 - 6 } & Instr. & Social & Afectivo & Moral & Política \\
\hline Nacional & 40.82 & 19.62 & 8.01 & 27.31 & 3.92 \\
Generacional & 37.84 & 17.27 & 27.63 & 17.27 & \\
Genero Masculino & 46.38 & 13.46 & 15.94 & 24.22 & \\
Genero Femenino & 33.77 & 18.76 & 25.16 & 22.29 & \\
Regional & 36.36 & 12.73 & 19.09 & 28.93 & 2.73 \\
Familiar & 29.68 & 20.8 & 22.46 & 26.77 & 0.28 \\
Personal & 37.08 & 6.18 & 33.15 & 23.59 & \\
\hline
\end{tabular}

En el nivel generacional las categorías predominantes son las instrumentales y afectivas, lo que señala un componente importante de involucramiento personal con la condición de «joven», que a su vez se va repetir en nivel familiar. En el nivel de género es importante señalar las diferencias en cuanto al género masculino y femenino, encontrándose un mayor equilibrio en las respuestas referidas al género femenino, por cuanto las categorías instrumentales, afectivas y morales están más proporcionalmente distribuidas si lo comparamos con las respuestas respecto al género masculino, cuyo predominio es instrumental y moral. Las respuestas correspondientes a la categoría afectiva son menores, lo que a su vez indica una tendencia a la asociación más directa de la afectividad con la mujer y más bien un distanciamiento de la afectividad con respecto al hombre.

En el nivel regional las categorías predominantes son las instrumentales y morales y secundariamente afectivas, lo que estaría indicando que en la percepción del «limeño» hay una presencia valorativa de sus respuestas.

En el nivel familiar es donde se encuentra una mayor homogeneidad en las respuestas 
distribuyéndose éstas en forma casi proporcional en las categorías instrumentales, afectivas, sociales y morales con excepción de la casi ausencia de respuestas en la categoría política. La homogeneidad que se encuentra en las respuestas respecto a las categorías señaladas guardan relación con la tendencia al componente positivo predominante en el nivel familiar.

En el nivel personal el predominio es de las categorías instrumentales y afectivas, respuestas muy parecidas a las del nivel generacional, donde también ambas categorías son las predominantes.

En la tabla No. 2 se observan los resultados de cada uno de los niveles de identidad considerando la orientación positiva o negativa de las respuestas agrupadas en cada una de las cinco categorías. En cuanto a la identidad nacional se observa que es la única dimensión donde predominan las respuestas negativas (55.41) sobre las positivas (43.49), lo que indica una tendencia constante en el conjunto de Países Latinoamericanos, que tienden a sobrevalorar lo negativo y desvalorizar lo positivo; pero por otra parte también se observa si se compara con las investigaciones previas (Montero, M. 1993) una reducción del componente negativo y una elevación del componente positivo, es así que la característica de flojos (110) es la que mayor frecuencia obtiene, el segundo lugar de mayor frecuencia es el de alegres, y el tercer lugar le corresponde a la característica trabajador (65) que tiene una orientación positiva, lo que es importante en la medida que se reconoce un aspecto que era totalmente opacado por la característica de ocioso o flojo.

La identidad generacional tiene un componente predominantemente positivo $(66.76 \%)$, y las características negativas ocupan una tercera parte del total de respuestas, lo interesante es que a diferencia de la percepción social negativa acerca de los jóvenes, éstos se perciben con características positivas donde predominan las respuestas tales como: libres, alegres, sociables, responsables, estudiosos, ubicados fundamentalmente en la categoría instrumental y afectiva.

En cuanto a la identidad de género se observa un predominio positivo tanto en hombres como en mujeres, siendo superior el porcentaje positivo en las mujeres que en los hombres, e incluso en las respuestas negativas, las mujeres tienen una menor presencia de las mismas, lo que es un dato novedoso, por cuanto la mujer es un sector de la población tradicionalmente marginado y hacia la cual se han desarrollado socialmente estereotipos negativos, por otra parte observamos como positivo el hecho que los hombres reconozcan características negativas de ellos como el machismo, el ser borrachos, agresivos. Reconocer lo negativo en su justa dimensión es un paso para el cambio.

En cuanto a la identidad que nosotros hemos denominado regional, referida a los limeños, también las características tienen una orientación prioritariamente positiva $(52.29 \%)$ y donde en el componente negativo aparece una característica no presente en otras dimensiones en la amplitud de frecuencia, que es la de ser racista, que implica un reconocimiento explícito de dicha actitud, probablemente porque a pesar de ser limeños, son hijos de migrantes que no sólo han sufrido sino sufren y son víctimas de prejuicio aún en la actualidad.

En cuanto a la identidad familiar es la dimensión con características más positivas que todas las demás, lo que estaría indicando que la familia pese a las crisis por las que atraviesa, todavía es una de las instituciones fundamentales en la vida de los jóvenes, sobre 
todo si se tiene en cuenta que es la única dimensión donde predominan las categorías afectivas y morales con una orientación positiva y donde la solidaridad, la alegría, la unión familiar, la responsabilidad y el amor son las características de mayor frecuencia.

La familia institución fundamental en la socialización de las personas, al parecer cumple un papel de soporte afectivo muy importante en los jóvenes.

En cuanto a la identidad personal, es la que ocupa el segundo lugar en porcentaje positivo, después de la identidad familiar, lo interesante es que el porcentaje de respuestas negativas es menor (17\%) y donde la categoría afectivas predominante en respuestas positivas, esto es importante sobre todo si se tiene en cuenta la edad de los sujetos (15-18 años) que generalmente es muy contradictoria desde el punto de vista afectivo.

Incluso características que socialmente se piensa son ajenas al joven, son señaladas por ellos en un sentido positivo, remarcando los sentimientos de amistad y amor y la consideración de ser una buena persona.

Tabla $\mathbf{N}^{0} 2$

Distribución de respuestas por valoración positiva y negativa en categorías y niveles de identidad en porcentajes

\begin{tabular}{|l|c|c|c|c|c|c|c|c|c|c|}
\hline \multirow{2}{*}{\multicolumn{1}{|c|}{ Identidad }} & \multicolumn{10}{|c|}{ Categorías } \\
\cline { 2 - 11 } & \multicolumn{2}{|c|}{ Instrucc. } & \multicolumn{2}{c|}{ Social } & \multicolumn{2}{c|}{ Afectivo } & \multicolumn{2}{c|}{ Moral } & \multicolumn{2}{c|}{ Política } \\
\cline { 2 - 10 } & + & - & + & - & + & - & + & - & + & - \\
Nacional & 15.23 & 25.59 & 5.02 & 14.6 & 5.34 & 2.67 & 14.91 & 12.4 & 3.45 & 0.47 \\
Generacional & 24.46 & 13.38 & 11.51 & 5.76 & 25.47 & 2.16 & 8.35 & 8.92 & & \\
Genero Masculino & 35.61 & 10.77 & 5.80 & 7.66 & 12.21 & 3.73 & 15.32 & 8.90 & & \\
Genero Femenino & 28.03 & 5.74 & 14.35 & 4.41 & 24.06 & 1.10 & 15.45 & 6.84 & & \\
Regional & 27.27 & 9.09 & 9.85 & 2.88 & 18.79 & 0.3 & 12.42 & 16.51 & 2.73 & \\
Familiar & 21.64 & 8.04 & 12.2 & 8.6 & 21.91 & 0.55 & 23.58 & 3.19 & 0.28 & \\
Personal & 26.72 & 10.36 & 5.22 & 0.96 & 30.11 & 3.04 & 20.63 & 2.96 & & \\
\hline
\end{tabular}

\section{CONCLUSIONES}

1. La identidad en sus diferentes niveles se diferencia por su orientación positiva y negativa, así como por el predominio de determinadas categorías.

2. La identidad nacional tiene un predominio de características negativas, siendo las categorías instrumentales y morales las de mayor incidencia negativa.

3. La identidad nacional tiene en sus características positivas componentes importantes como el ser trabajador, ser alegre, buena persona y solidario.

4. La identidad generacional es altamente positiva en contraste con la percepción social negativa respecto a los jóvenes.

5. La identidad de género es positiva tanto para hombres como para mujeres. pero la identidad femenina tiene componentes más positivos y menor presencia de componentes 
negativos, a diferencia de los hombres que tienen el doble de características negativas en relación a las mujeres.

6. La identidad de los limeños es positiva; pero reconoce características negativas como: la ociosidad, el conformismo, el racismo y la irresponsabilidad, lo que implica un cierto grado de conciencia de dichas problemáticas.

7. La identidad familiar es la que tiene características más altamente positivas que todos los otros niveles de identidad, y se reconoce en la misma características categorizadas como morales y afectivas.

8. La identidad personal también es positiva, y tiene una menor proporción de componentes negativos, y donde los componentes positivos de la categoría afectiva son los predominantes.

\section{BIBLIOGRAFIA}

Aceituno, Roberto (1989) Anomia y alienación en jóvenes de 4to. de media en Santiago de chile. En memorias del XXII Congreso Interamericano de Psicología: Buenos Aires.

Bustamante, R. (1986) Raza e identidad positiva y negativa en Lima. En Psicología y realidad peruana. Mosca Azul: Lima.

Cornejo Polar A. (1981) La cultura nacional, problema y posibilidad. Lima: Lluvia editores.

Flores Galindo, A. (1988) La imagen y el espejo. Revista márgenes N* 4, 55-85 : Lima.

Gissi, J. (1987) Identidad latinoamericana Santiago de Chile: Andes.

Gutierrez, F. (1985) Nación, nacionalidad y nacionalismo. Barcelona: Salval.

Herencia, C. (1989) Identidad Social y comportamiento político en el Perú. Memorias del XXII Congreso Interamericano de Psicología. Buenos Aires.

Montero, M. (1987) Psicología política latinoamericana. Venezuela: Panapo.

Montoya R. (1980) Identidad, percepción campesina y problema nacional. Revista Tarea $\mathrm{N}^{\mathrm{o}} 138$.

Portocarrero, J. y Oliart P. (1989) El Perú desde la escuela. Lima: Instituto de apoyo agrario.

Portocarrero, G. (1993) Racismo y mestizaje. Lima: Casa Sur

Salazar, J. et. Al (1986) Bases psicológicas del nacionalismo. México: Trillas. 